\title{
Targeting Fibroblast Growth Factor Pathways in Prostate Cancer
}

\author{
Paul Corn ${ }^{1}$, Fen Wang ${ }^{2}$, Wallace L. McKeehan ${ }^{2}$, and Nora Navone ${ }^{1}$ \\ ${ }^{1}$ The University of Texas, MD Anderson Cancer Center \\ ${ }^{2}$ Institute of Biosciences and Technology, Texas A\&M Health Science Center
}

\section{Abstract}

\begin{abstract}
Advanced prostate cancer carries a poor prognosis and novel therapies are needed. Research has focused on identifying mechanisms that promote angiogenesis and cellular proliferation during prostate cancer progression from the primary tumor to bone- the principal site of prostate cancer metastases. One candidate pathway is the fibroblast growth factor (FGF) axis. Aberrant expression of FGF ligands and FGF receptors leads to constitutive activation of multiple downstream pathways involved in prostate cancer progression, including mitogen-activated protein kinase, phosphoinositide 3-kinase, and phospholipase $\mathrm{C} \gamma$. The involvement of FGF pathways in multiple mechanisms relevant to prostate tumorigenesis s provides a rationale for the therapeutic blockade of this pathway, and two small-molecule tyrosine kinase inhibitors-dovitinib and nintedanibare currently in phase 2 clinical development for advanced prostate cancer. Preliminary results from these trials suggest that FGF pathway inhibition represents a promising new strategy to treat castrate-resistant disease.
\end{abstract}

\section{Keywords}

fibroblast growth factor; metastatic castrate-resistant prostate cancer; tyrosine kinase inhibitors; dovitinib; nintedanib

\section{Introduction}

Prostate cancer is the most commonly diagnosed malignancy in males and the second most deadly, with approximately 241,740 new cases and 28,170 deaths reported in the United States in 2012(1). Although most cases detected early using prostate-specific antigen screening are curable with local therapies, metastatic disease remains incurable. Metastatic disease is initially treated very effectively with androgen-deprivation therapy but most patients will eventually develop castrate resistance, defined as disease progression that occurs despite serum levels of testosterone $<50 \mathrm{ng} / \mathrm{dL}$. Patients with metastatic castrateresistant prostate cancer (mCRPC) carry a poor prognosis, with a median overall survival of approximately 14 months(2).

Although docetaxel chemotherapy remains a standard treatment option for mCRPC, several new agents have recently been approved. Sipuleucel-T, a cancer vaccine, is indicated for asymptomatic or minimally symptomatic mCRPC. Abiraterone acetate and enzalutamide inhibit intracrine androgen receptor (AR) signaling associated with castrate resistance. Both

Contact Information: Paul G. Corn, Department of Genitourinary Medical Oncology, The University of Texas MD Anderson Cancer Center, Box 1374, 1515 Holcombe Boulevard, Houston, Texas 77030, Phone: (713) 563-7204, Fax: (713) 563-0993, pcorn@mdanderson.org.

Conflict of Interest Information: PGC has served on a Novartis-sponsored advisory board for Dovitnib but did not receive any financial compensation. 
are indicated after docetaxel therapy, and abiraterone is now available for chemotherapynaive patients. Cabazitaxel, a taxane analog, is approved after docetaxel failure. Although these agents have each positively affected survival, mCRPC remains incurable and new therapies are needed (3).

Research efforts elucidating signaling pathways and cellular processes involved in prostate cancer progression have produced many therapies currently in clinical development, including immunomodulators, inhibitors of intracrine AR signaling, and small-molecule inhibitors of oncogenic pathways (4). One such pathway is the fibroblast growth factor (FGF) axis, which is required for normal prostate and skeletal development but becomes aberrantly activated in prostate cancer $(5,6)$. This review discusses the role of FGF signaling in prostate cancer and the potential for FGF inhibition as a rational therapy strategy in advanced disease.

\section{Fibroblast growth factor signaling pathways}

FGF pathway components are ubiquitously expressed and play important roles in diverse biologic processes including embryonic and organ development, wound healing, and carcinogenesis. The FGF axis and its downstream pathways regulate many mechanisms including mitogenesis, differentiation, angiogenesis, survival, and motility/ invasiveness(5,6). FGF ligands comprise a gene family consisting of 22 structurally related proteins that are further divided into 7 subfamilies based on sequence homology: FGF1-2, FGF4-6, FGF3/7/10/22, FGF8/17/18, FGF9/16/20, FGF11-14, and FGF19/21/23(6). FGF 11-14 ligands do not function as ligands, and FGF 19/21/23 ligands are endocrine messengers. The remaining FGFs signal through autocrine or paracrine mechanisms by activating FGF receptors (FGFRs) in partnership with heparan sulfate proteoglycans (HSPs) $(7,8)$. The FGFR is a single glycosylated polypeptide chain that has an extracellular ligandbinding domain, a transmembrane domain, and an intracellular tyrosine kinase domain. There are 4 recognized FGFRs (FGFR1, $-2,-3$, and -4 ) that function as receptor tyrosine kinases. Four highly conserved genes encode an extensive repertoire of alternatively spliced variants of FGFR that vary in both the extracellular ligand-binding and intracellular kinase domains $(8,9)$. The extracellular domain comprises three immunoglobulin-like motifs, the third of which has differing specificities for various FGF ligands(10). FGFR1, FGFR2, and FGFR3 encode two versions (IIIb and IIIc) of immunoglobulin-like domains in mutually exclusive exons. The IIIb and IIIc isoforms are mainly expressed in epithelial and mesenchymal tissues, respectively, and have different ligand selectivities $(11,12)$. Despite the diversity of ligands, FGFR variants and oligosaccharide motifs that make up the FGFR signaling complex, cell-specific expression of isoforms, and the combination of the three individual components confer specificity on intercompartmental signaling. Furthermore, the partitioning of FGF ligand and FGFR isotypes, combined with the cell-type specificity of HSP, creates directionally specific paracrine communication from one cell type or compartment to another.

A schematic of FGF/FGFR signaling is shown in Figure 1. Current models suggest a symmetric dimeric complex of two FGFs, two FGFRs, and two HSP chains. Activation triggers autophosphorylation of FGFR followed by FGFR substrate 2 (FRS2)

phosphorylation and recruitment of the docking proteins FRS2 and phospholipase-C gamma $(\mathrm{PLC} \gamma)(13,14)$. FRS2 has two isoforms (FRS2 $\alpha$ and FRS2 $\beta$ ) that largely mediate FGFR signaling. FRS2a has multiple tyrosine and serine/threonine phosphorylation sites that are phosphorylated upon ligand-induced FGFR activation. These phosphorylation sites constitute binding sites for recruitment and activation of additional adaptors and signaling molecules, such as Shp2 and growth factor receptor-bound 2, which link to intracellular networks, most commonly the mitogen-activated protein kinase (MAPK/ERK) and PI3K/ 
AKT pathways $(15,16)$. Activated ERK1/2 stimulates transcription factors such as the ETS family of proteins, leading to alterations in cellular gene expression. Several other pathways are also activated by FGFRs, depending on the cellular context, including the p38 MAPK and Jun N-terminal kinase pathways, signal transducer and activator of transcription signaling, and ribosomal protein S6 kinase 2. It should be noted, however, that other important growth factor ligand-receptor pathways (e.g. IL-6, EGF, TGF- $\beta$ ) signal through molecular intermediaries (e.g. SOS, RAS) shared with FGF/FGFR activation, so cell fate is ultimately complex.

The FGF/FGFR signaling cascade is regulated by a number of positive and negative modulators (Fig.1). Secreted FGF binding protein (SFBP) can reversibly bind FGF1 and FGF2, releasing them from the extracellular matrix to stimulate signaling(17). The Sprouty (Spry) protein family is a feedback regulator of the FGF pathway at the posttranslational level (18). Tyrosine phosphorylation of Sprys creates a decoy site that binds the docking molecule growth factor receptor-bound 2 and prevents Son of Sevenless from activating the Ras-MAPK pathway or by directly binding to RAF and blocking subsequent MAPK signaling(19-21). Furthermore, similar expression to FGF (SEF) family members and MAPK phosphatase 3 (MKP3) are attenuators of the FGF-activated downstream MAPK pathway. Thus, FGF/FGFR signaling is regulated differently based on the specific FGF ligand, the FGFR splice variant expressed, and the presence and activity of regulatory factors in a tissue- and cellular-specific context (22).

\section{FGF signaling in human cancer}

Aberrant activation of FGF/FGFR signaling is common in many epithelial cancers including hepatocellular carcinoma, melanoma, and lung, breast, bladder, endometrial, head and neck, and prostate cancers (23). There are multiple mechanisms for dysregulation that occur principally through alterations of FGFRs rather than FGFs (23). One mechanism is ligandindependent activation of FGFR via point mutations that promote dimerization or enhance kinase activity. For example, a point mutation in FGFR3 has been detected in 50\% to 60\% of non-muscle invasive urothelial carcinomas. A second mechanism is FGFR gene amplification and overexpression, which result in excessive signaling. For example, amplification of chromosomal region 8p11-12, which encompasses FGFR1, is present in approximately $10 \%$ of breast carcinomas (23). A third mechanism involves an alternatively spliced FGFR variant with altered ligand selectivity that triggers inappropriate signaling activation (24). In contrast, mutations in FGF ligands appear to be rare in human cancers (23). Last, FGF/FGFR-mediated oncogenesis may also be associated with a failure to attenuate signaling resulting from loss of function of negative regulators such as Spry(25).

\section{FGF signaling in prostate cancer}

Development and homeostasis of the normal prostate gland depends on androgenic stimulation and epithelial-mesenchymal interactions $(26,27)$. Mesenchymal tissues secrete paracrine factors that stimulate epithelial maintenance and growth, and FGF ligands are key messengers(26). FGF10 is expressed in the mesenchyme and is required for prostate growth and development, as evidenced by developmental defects in prostate precursor buds in an FGF10 knockout mouse(28). Studies have also identified biologically relevant levels of FGF2, FGF7, and FGF9 in normal prostate mesenchyme (fibroblasts and/or endothelial cells), whereas FGFRs with specificity for these ligands are expressed in secretory prostatic epithelium(25). FGF10 and FGF7 may be partially redundant, as evidenced by the development of a viable male reproductive system in an FGF7 knockout mouse(29). FGFR2 was shown to be required for prostate organogenesis and the acquisition of androgen 
dependency for tissue homeostasis using a conditional knockout mouse (30). Thus, FGF/ FGFR signaling is necessary for development and homeostasis of the normal prostate gland.

Aberrant FGF signaling has been implicated in PCa development and progression (31-34). With respect to early prostate cancer, multiple studies have demonstrated involvement of dysregulated FGF/FGFR signaling in all developmental stages of prostatic intraepithelial neoplasia (PIN) - carcinoma in situ of the prostate characterized by initial cell proliferation and anaplasia - followed by invasive carcinoma, angiogenesis, and metastasis. For example, forced expression of constitutively active mutant of FGFR1 leads to development of highgrade PIN lesions (35). Perturbations of FGF/FGFR pathway signaling also contribute to the development of PIN and prostate cancer through mechanisms that mimic the developmental program (36).

Clear recent evidence that paracrine activation of FGFR in prostate epithelial cells leads to PIN or prostate cancer was provided using a tissue recombination prostate regeneration system (37)(Fig 2). In this model, adult dissociated normal mouse prostate epithelial cells (mNPE) are combined with embryonic urogenital sinus mesenchyme (UGSM) and grafted under the kidney capsule of a SCID mouse, resulting in formation of prostate gland-like structures. This model allows for genetic manipulation of the epithelial and mesynchymal compartments independently to study effects of paracrine factors on adult prostate epithelial cells. In this system, mesenchymal expression of FGF10 leads to FGFR1 dependent PIN or prostate cancer development and enhanced AR expression in the neoplastic epithelium (Fig 2). Inhibition of epitelial FGFR1 signaling using a dominant negative FGFR1 led to reversal of the cancer phenotype.

In addition, altered expression of FGFR1, but not of FGFR2, has been shown to induce PIN in the juxtaposition of chemical inducers of dimerization and kinase (JOCK)-1 and -2 transgenic mouse models, respectively (38)(Fig 3a). JOCK-1 mice subsequently develop invasive prostate carcinoma and metastasis. Furthermore, conditional deletion of FRS2a in the mouse prostate inhibited the initiation and progression of prostate cancer induced by oncogenic viral proteins (Fig 3b) (39)Conditional inactivation of FGFR1 also impairs development of PIN, prostate cancer progression, and metastasis in the TRAMP model(40). Together, these reports suggest that activation of FGFR1 signaling is sufficient to induce prostate cancer development and progression in mouse models of prostate cancer.

It has also been shown that increased production of FGF ligands by prostatic secretory epithelial cells induces autocrine signaling and independence from stromal regulation, stimulating aberrant epithelial growth and cellular dysplasia (26,27). For example, FGF8 and its cognate receptors FGFR1IIIc and FGFR2IIIc are overexpressed in human samples of PIN and prostate cancer compared with controls (41). Also, the FGF9-FGFR3 axis has been reported to signal from neoplastic epithelium to mesenchyme in Dunning R3327 rat prostate cancer models (42).

With respect to later stages of prostate cancer progression, multiple studies have demonstrated involvement of aberrant FGF/FGFR signaling in driving epithelialmesenchymal transition (EMT). EMT is a process whereby polarized epithelial cells lose epithelial characteristics and acquire mesenchymal features, including enhanced migratory capacity, invasiveness, and elevated resistance to apoptosis(43). EMTs normally occur during embryonic development and wound healing/tissue regeneration, but the mechanism is coopted during the acquisition of malignancy. In JOCK-1 mice, inducible ectopic FGFR1 induced the formation of tumors that possessed mesenchymal-like cells with residual epithelial hallmarks that were presumed to be remnants of EMT (38). Also of note, shifts in 
alternative splicing of FGFR1 and FGFR2 from IIIb (epithelial) to IIIc (mesenchymal) isoforms are associated with EMT in prostate and other types of cancer(24).

In support of the aforementioned data, upregulation of FGF family members in primary prostate cancer is positively associated with higher grades of cancer and clinical stage (44, 45). FGFs have been shown to be overexpressed predominantly in epithelial cells (FGF1, FGF6, FGF8, FGF17), stromal fibroblasts (FGF2) or both (FGF7), supporting the existence of autocrine-paracrine growth promoting effects(25). Furthermore, FGF8 is expressed at low levels in normal prostate but its overexpression in prostate cancer (46-48) is associated with decreased patient survival(45). Mechanistic studies have established links between some ligands and prostate cancer. For example, attenuating FGF2 activity inhibits cell proliferation, migration, and invasion in cell culture $(49,50)$, whereas FGF2 knockout in a transgenic mouse model of prostate adenocarcinoma inhibited tumor progression (51). In addition, exogenous FGF1 induces expression of matrix metalloproteinases, which are suggested to play a role in tumor metastasis, in prostate cancer LNCaP cells (52).

Dysregulated expression of FGFRs has also been associated with prostate cancer. Overexpression of FGFR1 has been found in prostate tumors (53). Additionally, ectopic expression of FGFR1 accelerated tumorigenicity of rat premalignant prostate epithelial cells (33). In contrast, FGFR2 appears to have the opposite effect, whereby its downregulation is associated with neoplastic progression $(33,54)$. Furthermore, reduced levels of "similar expression to FGF"- an FGF pathway inhibitor-is correlated with higher grades of cancer (44), demonstrating that oncogenic dysregulation of the FGF axis can occur via multiple mechanisms.

\section{Rationale for FGF inhibition in prostate cancer}

FGF/FGFR signaling is intimately involved in promoting angiogenesis, involving potential crosstalk with platelet-derived growth factor (PDGF) and vascular endothelial growth factor (VEGF) pathways. In the normal prostate, FGF2 is a known regulator of angiogenesis (55), and a correlation between FGF8 and VEGF expression exists in prostate cancer (56). Additionally, conditional FGFR1 expression via the JOCK-1 model induced angiogenesis through upregulation of hypoxia inducible factor 1a, VEGF, and angiogenin-2 and downregulation of angiogenin-1(57). Osteoblasts can also promote angiogenesis through DJ-1 - a soluble factor that induces angiogenesis in endothelial cells via activation of FGFR1 (58). The FGF pathway is also known to function as a resistance mechanism for anti-angiogenic therapy in preclinical models for other tumor types(59). These data highlight FGF/FGFR signaling as a driver of angiogenesis in prostate cancer and provide a rationale for dual FGF/VEGFR inhibition as therapy(59).

FGFs also have a role in the development of bone metastases, which occur in approximately $80 \%$ of patients with advanced prostate cancer(60). These metastases often abnormally express FGF8 and/or FGF9, which promote osteoblast proliferation/differentiation in culture $(61,62)$. It has also been shown that exogenous expression of FGF8 forms bone lesions and spurs the growth of intratibial tumors in a model simulating bone metastasis of prostate cancer (63) and that FGF9 mediates the osteoblastic progression of human prostate cancer cells in the bone of mice(61). Additional crosstalk between bone stroma and prostate cancer cells mediated by hedgehog and bone morphogenic protein signaling may promote cancer survival through upregulation of FGF2 (64). A model of the principal FGFs and FGRs involved in prostate cancer progression is shown in Figure 4a. Therefore, inhibiting the FGF pathway could reduce the propensity of prostate tumors to metastasize and/or survive in bone. 
Lastly, the development of castrate resistance is associated with intracrine AR signaling and data from preclinical models, primary tumors, and metastatic tumors suggest that FGF and AR signaling are tightly intertwined (26). For example, de novo expression of AR in PC3 prostate cancer cells may upregulate FGF signaling by increasing the utilization of FGF2 (65). FGF7 and FGF8, which are normally induced by androgens, are both expressed in primary castrate-resistance tumors $(45,66)$, and the inhibition of FGF8 and FGF9 signaling has an antitumor effect in castrate-resistant mouse models(61, 67). Furthermore, the bone marrow microenvironment supports the development of castrate-resistant growth $(61,68)$, and bone biopsies from patients with mCRPC overexpress FGF9 more frequently than do primary tumors (56). FGF has also been linked to chemoresistance to multiple classes of agents, including taxanes $(69,70)$. Since, castrate-resistant tumors are frequently treated with taxane-based chemotherapy, a rationale exists to combine FGF inhibitors and chemotherapy in patients with mCRPC. Taken together, these results suggest that targeting the FGF pathway could have a clinical impact on CRPC in primary prostate tumors and metastatic disease (Fig. 4b).

\section{FGF pathway inhibition in prostate cancer}

A number of targeted agents that inhibit FGF/FGFR signaling now exist (Table 1). These agents include FGFR tyrosine kinase inhibitors (TKIs), monoclonal antibodies, and an FGF ligand trap. A potential advantage of TKIs with a broader spectrum of inhibition is their ability to target FGFR simultaneously with other angiogenic receptor tyrosine kinases, such as VEGFR and PDGFR. TKIs that target FGFRs along with other kinases include dovitinib, nintedanib (BIBF 1120), masitinib, lenvatinib, brivanib, orantinib, and PD173074 (5, 71). The first 6 agents are in phase 3 development for non-prostate tumors, including gastrointestinal stromal tumors, multiple myeloma, melanoma, renal cell carcinoma, hepatocellular carcinoma, and pancreatic, thyroid, and ovarian cancers (Table 1). Importantly, dovitinib and nintedanib are also being investigated in mCRPC.

Dovitinib is a small-molecule TKI that targets multiple kinases, including FGFR1, FGFR3, VEGFR1-3, PDGFR $\beta$, fms-related tyrosine kinase-3, and c-KIT. Preclinical studies have demonstrated upregulation of the tumor suppressor proteins p21 and p27 after dovitinib treatment in cell culture models of prostate cancer(72). On the basis of findings in an ARnegative prostate cancer xenograft model that suggested a role for FGF/FGFR in osteoblastic progression of CRPC in bone (61), a phase 2 trial of dovitinib in patients with bone mCRPC is currently underway (ClinicalTrials.gov: NCT00831792). Of 23 patients evaluable for response (> 1 cycle on therapy), 6 of 23 (26\%) experienced improvements on bone scan (1 complete response and 3 partial responses [PRs]) or in soft tissue metastases (2 PRs) at 8 weeks, with a median treatment duration of 19.9 weeks (range, 10-35 weeks). Thirteen of $23(57 \%)$ patients experienced stable disease at 8 weeks, with a median treatment duration of 11.7 weeks (range, 6-31 weeks), and 4 of 23 (17\%) experienced disease progression. Toxicities were mostly grade 1 or 2 . Grade 3 toxicities included fatigue, rash, and generalized weakness (each found in $<5 \%$ of the patients) and there were no Grade 4 toxicities (73).

Nintedanib (BIBF 1120)—a TKI that inhibits FGFR1-3, VEGFR1-3, PDGFRa and - $\beta$, Src, Lck, and Lyn - has shown preliminary activity in a phase 2 study in patients with mCRPC, yielding a PR rate of $4.3 \%$ and a stable disease rate of $30.4 \%(74)$. In a phase 1 trial, a combination of nintedanib, docetaxel, and prednisone produced a $\geq 50 \%$ reduction from baseline in prostate-specific antigen levels in $68.4 \%$ of 19 evaluable patients and a PR in 1 of 6 patients with measurable lesions(75). 
The observation that some TKIs have dose-limiting "off target" toxicities has spurred the development of more selective pan-FGFR inhibitors. AZD4547, AZD8010, and BGJ398 potently inhibit FGFR1-3, whereas AZD8010 also inhibits FGFR4, albeit to a lesser degree (76-78). AZD4547 is undergoing investigation in a phase 1 trial of advanced solid malignancies (NCT00979134). BGJ398 is being evaluated in a phase 1 trial in patients with advanced solid tumors harboring amplified FGFR1 or FGFR2 genes or mutated FGFR3(78). AZD8010 is still in preclinical development.

FGF signaling may also be more specifically abrogated by blocking ligand-receptor binding using monoclonal antibodies or a ligand trap. Three monoclonal antibodies that block FGF signaling are currently in development: MFGR1877S, GP369, and 1A6. MFGR1877S is a monoclonal antibody against FGFR3 (79) that is currently undergoing phase 1 testing for patients with advanced solid tumors, including those with CRPC (NCT01363024). Both GP369 and 1A6 are currently in preclinical development (80, 81). GP369 specifically blocks the IIIb splice variant of FGFR2, and 1A6 targets the ligand FGF19. HGS1036 (FP-1039) is a construct comprising the extracellular domain of FGFR1c fused with the Fc portion of IgG1 and is expected to be a decoy for FGF ligands having affinity for FGFR1c. A phase 1 study of HGS1036 in patients with metastatic or locally advanced unresectable solid tumors has been completed. A preliminary report documented a patient with prostate cancer who had tumor shrinkage(82), and final results are anticipated shortly. These agents have the potential to clinically affect mCRPC, and further investigation is expected.

\section{Conclusions and future directions}

An enlarging body of evidence supports a role for FGF/FGFR signaling in prostate cancer. Dysregulated signaling is associated with the development of PIN, EMT, and angiogenesis - critical biologic processes involved in cancer progression and metastasis. FGF/FGFR signaling between bone stroma and prostate cancer cells also promotes the formation of bone metastases and the emergence of castrate-resistant disease. Evidence from preclinical and clinical studies suggest that inhibitors of FGF/FGFR signaling warrant further investigation as a rational therapeutic strategy for the treatment of patients with advanced prostate cancer.

\section{Acknowledgments}

The authors acknowledge medical editorial assistance from Melanie Vishnu, PhD. Medical editorial assistance was funded by Novartis Pharmaceuticals.

Financial Support: The authors are supported by NIH grants CA096824 (FW) and the Prostate cancer SPORE grant 5P50 CA140388 (N.M.N. and P.G.C. F.W. and W.L. M.); by the Prostate Cancer Foundation (N.M.N. and P.G.C.); by the Cancer Prevention \& Research Institute of Texas grant RP11055 (N.M.N., F.W. and W.L. M.) and by the David H. Koch Center for Applied Research in Genitourinary Cancers at The University of Texas MD Anderson Cancer Center (N.M.N. and P.G.C.), Houston, TX.

NN has received a research grant from Astra Zeneca to study AZD4547.

\section{References}

1. American, Cancer, Society. Cancer facts and figures 2012. 2012

2. Kirby M, Hirst C, Crawford ED. Characterising the castration-resistant prostate cancer population: a systematic review. Int J Clin Pract. 2011; 65:1180-1192. [PubMed: 21995694]

3. Clarke JM, Armstrong AJ. Novel therapies for the treatment of advanced prostate cancer. Curr Treat Options Oncol. 2013; 14:109-126. [PubMed: 23322116]

4. Agarwal N, Sonpavde G, Sternberg CN. Novel molecular targets for the therapy of castrationresistant prostate cancer. Eur Urol. 2012; 61:950-960. [PubMed: 22209376] 
5. Daniele G, Corral J, Molife LR, de Bono JS. FGF receptor inhibitors: role in cancer therapy. Curr Oncol Rep. 2012; 14:111-119. [PubMed: 22311684]

6. Beenken A, Mohammadi M. The FGF family: biology, pathophysiology and therapy. Nat Rev Drug Discov. 2009; 8:235-253. [PubMed: 19247306]

7. Ornitz DM. FGFs, heparan sulfate and FGFRs: complex interactions essential for development. Bioessays. 2000; 22:108-112. [PubMed: 10655030]

8. McKeehan WL, Wang F, Kan M. The heparan sulfate-fibroblast growth factor family: diversity of structure and function. Prog Nucleic Acid Res Mol Biol. 1998; 59:135-176. [PubMed: 9427842]

9. Powers CJ, McLeskey SW, Wellstein A. Fibroblast growth factors, their receptors and signaling. Endocr Relat Cancer. 2000; 7:165-197. [PubMed: 11021964]

10. Ornitz DM, Xu J, Colvin JS, McEwen DG, MacArthur CA, Coulier F, et al. Receptor specificity of the fibroblast growth factor family. J Biol Chem. 1996; 271:15292-15297. [PubMed: 8663044]

11. Chellaiah AT, McEwen DG, Werner S, Xu J, Ornitz DM. Fibroblast growth factor receptor (FGFR) 3. Alternative splicing in immunoglobulin-like domain III creates a receptor highly specific for acidic FGF/FGF-1. J Biol Chem. 1994; 269:11620-11627. [PubMed: 7512569]

12. Johnson DE, Lu J, Chen H, Werner S, Williams LT. The human fibroblast growth factor receptor genes: a common structural arrangement underlies the mechanisms for generating receptor forms that differ in their third immunoglobulin domain. Mol Cell Biol. 1991; 11:4627-4634. [PubMed: 1652059]

13. Gotoh N. Regulation of growth factor signaling by FRS2 family docking/scaffold adaptor proteins. Cancer Sci. 2008; 99:1319-1325. [PubMed: 18452557]

14. Mohammadi M, Honegger AM, Rotin D, Fischer R, Bellot F, Li W, et al. A tyrosinephosphorylated carboxy-terminal peptide of the fibroblast growth factor receptor $(\mathrm{Flg})$ is a binding site for the SH2 domain of phospholipase C-gamma 1. Mol Cell Biol. 1991; 11:5068-5078. [PubMed: 1656221]

15. Kouhara H, Hadari YR, Spivak-Kroizman T, Schilling J, Bar-Sagi D, Lax I, et al. A lipid-anchored Grb2-binding protein that links FGF-receptor activation to the Ras/MAPK signaling pathway. Cell. 1997; 89:693-702. [PubMed: 9182757]

16. Choi SC, Kim SJ, Choi JH, Park CY, Shim WJ, Lim DS. Fibroblast growth factor-2 and-4 promote the proliferation of bone marrow mesenchymal stem cells by the activation of the PI3K-Akt and ERK1/2 signaling pathways. Stem Cells Dev. 2008; 17:725-736. [PubMed: 18788932]

17. Aigner A, Butscheid M, Kunkel P, Krause E, Lamszus K, Wellstein A, et al. An FGF-binding protein (FGF-BP) exerts its biological function by parallel paracrine stimulation of tumor cell and endothelial cell proliferation through FGF-2 release. International Journal of Cancer. 2001; 92:510-517.

18. Hacohen N, Kramer S, Sutherland D, Hiromi Y, Krasnow MA. sprouty encodes a novel antagonist of FGF signaling that patterns apical branching of the Drosophila airways. Cell. 1998; 92:253263. [PubMed: 9458049]

19. Casci T, Vinos J, Freeman M. Sprouty, an intracellular inhibitor of Ras signaling. Cell. 1999; 96:655-665. [PubMed: 10089881]

20. Hanafusa H, Torii S, Yasunaga T, Nishida E. Sprouty1 and Sprouty2 provide a control mechanism for the Ras/MAPK signalling pathway. Nat Cell Biol. 2002; 4:850-858. [PubMed: 12402043]

21. Kolch W. Coordinating ERK/MAPK signalling through scaffolds and inhibitors. Nat Rev Mol Cell Biol. 2005; 6:827-837. [PubMed: 16227978]

22. Turner N, Grose R. Fibroblast growth factor signalling: from development to cancer. Nat Rev Cancer. 2010; 10:116-129. [PubMed: 20094046]

23. Wesche J, Haglund K, Haugsten EM. Fibroblast growth factors and their receptors in cancer. Biochem J. 2011; 437:199-213. [PubMed: 21711248]

24. Holzmann K, Grunt T, Heinzle C, Sampl S, Steinhoff H, Reichmann N, et al. Alternative Splicing of Fibroblast Growth Factor Receptor IgIII Loops in Cancer. J Nucleic Acids. 2012; 2012:950508. [PubMed: 22203889]

25. Kwabi-Addo B, Ozen M, Ittmann M. The role of fibroblast growth factors and their receptors in prostate cancer. Endocr Relat Cancer. 2004; 11:709-724. [PubMed: 15613447] 
26. Lin Y, Wang F. FGF signalling in prostate development, tissue homoeostasis and tumorigenesis. Biosci Rep. 2010; 30:285-291. [PubMed: 20406196]

27. Cotton LM, O'Bryan MK, Hinton BT. Cellular signaling by fibroblast growth factors (FGFs) and their receptors (FGFRs) in male reproduction. Endocr Rev. 2008; 29:193-216. [PubMed: 18216218]

28. Donjacour AA, Thomson AA, Cunha GR. FGF-10 plays an essential role in the growth of the fetal prostate. Dev Biol. 2003; 261:39-54. [PubMed: 12941620]

29. Guo L, Degenstein L, Fuchs E. Keratinocyte growth factor is required for hair development but not for wound healing. Genes Dev. 1996; 10:165-175. [PubMed: 8566750]

30. Lin Y, Liu G, Zhang Y, Hu YP, Yu K, Lin C, et al. Fibroblast growth factor receptor 2 tyrosine kinase is required for prostatic morphogenesis and the acquisition of strict androgen dependency for adult tissue homeostasis. Development. 2007; 134:723-734. [PubMed: 17215304]

31. Yan G, Fukabori Y, Nikolaropoulos S, Wang F, McKeehan WL. Heparin-binding keratinocyte growth factor is a candidate stromal-to-epithelial-cell andromedin. Mol Endocrinol. 1992; 6:21232128. [PubMed: 1491693]

32. Yan G, Fukabori Y, McBride G, Nikolaropolous S, McKeehan WL. Exon switching and activation of stromal and embryonic fibroblast growth factor (FGF)-FGF receptor genes in prostate epithelial cells accompany stromal independence and malignancy. Mol Cell Biol. 1993; 13:4513-4522. [PubMed: 7687739]

33. Feng S, Wang F, Matsubara A, Kan M, McKeehan WL. Fibroblast growth factor receptor 2 limits and receptor 1 accelerates tumorigenicity of prostate epithelial cells. Cancer Res. 1997; 57:53695378. [PubMed: 9393762]

34. Lu W, Luo Y, Kan M, McKeehan WL. Fibroblast growth factor-10. A second candidate stromal to epithelial cell andromedin in prostate. J Biol Chem. 1999; 274:12827-12834. [PubMed: 10212269]

35. Wang F, McKeehan K, Yu C, Ittmann M, McKeehan WL. Chronic activity of ectopic type 1 fibroblast growth factor receptor tyrosine kinase in prostate epithelium results in hyperplasia accompanied by intraepithelial neoplasia. Prostate. 2004; 58:1-12. [PubMed: 14673947]

36. Schaeffer EM, Marchionni L, Huang Z, Simons B, Blackman A, Yu W, et al. Androgen-induced programs for prostate epithelial growth and invasion arise in embryogenesis and are reactivated in cancer. Oncogene. 2008; 27:7180-7191. [PubMed: 18794802]

37. Memarzadeh S, Xin L, Mulholland DJ, Mansukhani A, Wu H, Teitell MA, et al. Enhanced paracrine FGF10 expression promotes formation of multifocal prostate adenocarcinoma and an increase in epithelial androgen receptor. Cancer Cell. 2007; 12:572-585. [PubMed: 18068633]

38. Acevedo VD, Gangula RD, Freeman KW, Li R, Zhang Y, Wang F, et al. Inducible FGFR-1 activation leads to irreversible prostate adenocarcinoma and an epithelial-to-mesenchymal transition. Cancer Cell. 2007; 12:559-571. [PubMed: 18068632]

39. Zhang Y, Zhang J, Lin Y, Lan Y, Lin C, Xuan JW, et al. Role of epithelial cell fibroblast growth factor receptor substrate 2alpha in prostate development, regeneration and tumorigenesis. Development. 2008; 135:775-784. [PubMed: 18184727]

40. Yang F, Zhang Y, Ressler SJ, Ittmann MM, Ayala GE, Dang TD, et al. FGFR1 Is Essential for Prostate Cancer Progression and Metastasis. Cancer Res. 2013; 73:3716-2724. [PubMed: 23576558]

41. Valve EM, Nevalainen MT, Nurmi MJ, Laato MK, Martikainen PM, Harkonen PL. Increased expression of FGF-8 isoforms and FGF receptors in human premalignant prostatic intraepithelial neoplasia lesions and prostate cancer. Lab Invest. 2001; 81:815-826. [PubMed: 11406643]

42. Jin C, Wang F, Wu X, Yu C, Luo Y, McKeehan WL. Directionally specific paracrine communication mediated by epithelial FGF9 to stromal FGFR3 in two-compartment premalignant prostate tumors. Cancer Res. 2004; 64:4555-4562. [PubMed: 15231666]

43. Kalluri R, Weinberg RA. The basics of epithelial-mesenchymal transition. J Clin Invest. 2009; 119:1420-1428. [PubMed: 19487818]

44. Murphy T, Darby S, Mathers ME, Gnanapragasam VJ. Evidence for distinct alterations in the FGF axis in prostate cancer progression to an aggressive clinical phenotype. J Pathol. 2010; 220:452460. [PubMed: 19960500] 
45. Dorkin TJ, Robinson MC, Marsh C, Bjartell A, Neal DE, Leung HY. FGF8 over-expression in prostate cancer is associated with decreased patient survival and persists in androgen independent disease. Oncogene. 1999; 18:2755-2761. [PubMed: 10348350]

46. Gnanapragasam VJ, Robinson MC, Marsh C, Robson CN, Hamdy FC, Leung HY. FGF8 isoform b expression in human prostate cancer. Br J Cancer. 2003; 88:1432-1438. [PubMed: 12778074]

47. Leung HY, Dickson C, Robson CN, Neal DE. Over-expression of fibroblast growth factor-8 in human prostate cancer. Oncogene. 1996; 12:1833-1835. [PubMed: 8622905]

48. Tanaka A, Furuya A, Yamasaki M, Hanai N, Kuriki K, Kamiakito T, et al. High frequency of fibroblast growth factor (FGF) 8 expression in clinical prostate cancers and breast tissues, immunohistochemically demonstrated by a newly established neutralizing monoclonal antibody against FGF 8. Cancer Res. 1998; 58:2053-2056. [PubMed: 9605740]

49. Wesley UV, McGroarty M, Homoyouni A. Dipeptidyl peptidase inhibits malignant phenotype of prostate cancer cells by blocking basic fibroblast growth factor signaling pathway. Cancer Res. 2005; 65:1325-1334. [PubMed: 15735018]

50. Yang F, Strand DW, Rowley DR. Fibroblast growth factor-2 mediates transforming growth factorbeta action in prostate cancer reactive stroma. Oncogene. 2008; 27:450-459. [PubMed: 17637743]

51. Polnaszek N, Kwabi-Addo B, Peterson LE, Ozen M, Greenberg NM, Ortega S, et al. Fibroblast growth factor 2 promotes tumor progression in an autochthonous mouse model of prostate cancer. Cancer Res. 2003; 63:5754-5760. [PubMed: 14522896]

52. Udayakumar TS, Nagle RB, Bowden GT. Fibroblast growth factor-1 transcriptionally induces membrane type-1 matrix metalloproteinase expression in prostate carcinoma cell line. Prostate. 2004; 58:66-75. [PubMed: 14673954]

53. Sahadevan K, Darby S, Leung HY, Mathers ME, Robson CN, Gnanapragasam VJ. Selective overexpression of fibroblast growth factor receptors 1 and 4 in clinical prostate cancer. J Pathol. 2007; 213:82-90. [PubMed: 17607666]

54. Freeman KW, Gangula RD, Welm BE, Ozen M, Foster BA, Rosen JM, et al. Conditional activation of fibroblast growth factor receptor (FGFR) 1, but not FGFR2, in prostate cancer cells leads to increased osteopontin induction, extracellular signal-regulated kinase activation, and in vivo proliferation. Cancer Res. 2003; 63:6237-6243. [PubMed: 14559809]

55. Doll JA, Reiher FK, Crawford SE, Pins MR, Campbell SC, Bouck NP. Thrombospondin-1, vascular endothelial growth factor and fibroblast growth factor-2 are key functional regulators of angiogenesis in the prostate. Prostate. 2001; 49:293-305. [PubMed: 11746276]

56. West AF, O'Donnell M, Charlton RG, Neal DE, Leung HY. Correlation of vascular endothelial growth factor expression with fibroblast growth factor-8 expression and clinico-pathologic parameters in human prostate cancer. Br J Cancer. 2001; 85:576-583. [PubMed: 11506499]

57. Winter SF, Acevedo VD, Gangula RD, Freeman KW, Spencer DM, Greenberg NM. Conditional activation of FGFR1 in the prostate epithelium induces angiogenesis with concomitant differential regulation of Ang-1 and Ang-2. Oncogene. 2007; 26:4897-4907. [PubMed: 17297442]

58. Kim JM, Shin HI, Cha SS, Lee CS, Hong BS, Lim S, et al. DJ-1 promotes angiogenesis and osteogenesis by activating FGF receptor-1 signaling. Nat Commun. 2012; 3:1296. [PubMed: 23250426]

59. Casanovas O, Hicklin DJ, Bergers G, Hanahan D. Drug resistance by evasion of antiangiogenic targeting of VEGF signaling in late-stage pancreatic islet tumors. Cancer Cell. 2005; 8:299-309. [PubMed: 16226705]

60. Bubendorf L, Schopfer A, Wagner U, Sauter G, Moch H, Willi N, et al. Metastatic patterns of prostate cancer: an autopsy study of 1,589 patients. Hum Pathol. 2000; 31:578-583. [PubMed: 10836297]

61. Li ZG, Mathew P, Yang J, Starbuck MW, Zurita AJ, Liu J, et al. Androgen receptor-negative human prostate cancer cells induce osteogenesis in mice through FGF9-mediated mechanisms. J Clin Invest. 2008; 118:2697-2710. [PubMed: 18618013]

62. Valta MP, Hentunen T, Qu Q, Valve EM, Harjula A, Seppanen JA, et al. Regulation of osteoblast differentiation: a novel function for fibroblast growth factor 8. Endocrinology. 2006; 147:21712182. [PubMed: 16439448] 
63. Valta MP, Tuomela J, Bjartell A, Valve E, Vaananen HK, Harkonen P. FGF-8 is involved in bone metastasis of prostate cancer. Int J Cancer. 2008; 123:22-31. [PubMed: 18386787]

64. Nishimori H, Ehata S, Suzuki HI, Katsuno Y, Miyazono K. Prostate cancer cells and bone stromal cells mutually interact with each other through bone morphogenetic protein-mediated signals. J Biol Chem. 2012; 287:20037-20046. [PubMed: 22532569]

65. Rosini P, Bonaccorsi L, Baldi E, Chiasserini C, Forti G, De Chiara G, et al. Androgen receptor expression induces FGF2, FGF-binding protein production, and FGF2 release in prostate carcinoma cells: role of FGF2 in growth, survival, and androgen receptor down-modulation. Prostate. 2002; 53:310-321. [PubMed: 12430142]

66. Leung HY, Mehta P, Gray LB, Collins AT, Robson CN, Neal DE. Keratinocyte growth factor expression in hormone insensitive prostate cancer. Oncogene. 1997; 15:1115-1120. [PubMed: 9285567]

67. Maruyama-Takahashi K, Shimada N, Imada T, Maekawa-Tokuda Y, Ishii T, Ouchi J, et al. A neutralizing anti-fibroblast growth factor (FGF) 8 monoclonal antibody shows anti-tumor activity against FGF8b-expressing LNCaP xenografts in androgen-dependent and -independent conditions. Prostate. 2008; 68:640-650. [PubMed: 18213631]

68. Lescarbeau RM, Seib FP, Prewitz M, Werner C, Kaplan DL. In vitro model of metastasis to bone marrow mediates prostate cancer castration resistant growth through paracrine and extracellular matrix factors. PLoS One. 2012; 7:e40372. [PubMed: 22870197]

69. Gan Y, Wientjes MG, Au JL. Expression of basic fibroblast growth factor correlates with resistance to paclitaxel in human patient tumors. Pharm Res. 2006; 23:1324-1331. [PubMed: 16741658]

70. Song S, Wientjes MG, Gan Y, Au JL. Fibroblast growth factors: an epigenetic mechanism of broad spectrum resistance to anticancer drugs. Proc Natl Acad Sci U S A. 2000; 97:8658-8663. [PubMed: 10890892]

71. Mohammadi M, Froum S, Hamby JM, Schroeder MC, Panek RL, Lu GH, et al. Crystal structure of an angiogenesis inhibitor bound to the FGF receptor tyrosine kinase domain. EMBO J. 1998; 17:5896-5904. [PubMed: 9774334]

72. Vallo S, Mani J, Stastny M, Makarevic J, Juengel E, Tsaur I, et al. The prostate cancer blocking potential of the histone deacetylase inhibitor LBH589 is not enhanced by the multi receptor tyrosine kinase inhibitor TKI258. Invest New Drugs. 2013; 31:265-272. [PubMed: 22801803]

73. Wan X, Corn P, Yang J, Starbuck MW, Efstathiou E, Wang F, et al. Fibroblast growth factor receptor blockade in prostate cancer bone metastases. American Association for Cancer Research. Annual Meeting. 2013

74. Molife, R.; de Bono, JS.; Bell, S.; Lumsden, G.; Temple, G. A phase II trial to compare BIBF 1120 or BIBW 2992 monotherapy versus a combination of sequential administration of both medications in patients with hormone refractory prostate cancer (HRPC); ASCO Genitourinary Cancers Symposium; 2009.

75. Bousquet G, Alexandre J, Le Tourneau C, Goldwasser F, Faivre S, de Mont-Serrat H, et al. Phase I study of BIBF 1120 with docetaxel and prednisone in metastatic chemo-naive hormone-refractory prostate cancer patients. Br J Cancer. 2011; 105:1640-1645. [PubMed: 22027711]

76. Feng S, Shao L, Yu W, Gavine P, Ittmann M. Targeting fibroblast growth factor receptor signaling inhibits prostate cancer progression. Clin Cancer Res. 2012; 18:3880-3888. [PubMed: 22573348]

77. Gavine PR, Mooney L, Kilgour E, Thomas AP, Al-Kadhimi K, Beck S, et al. AZD4547: an orally bioavailable, potent, and selective inhibitor of the fibroblast growth factor receptor tyrosine kinase family. Cancer Res. 2012; 72:2045-2056. [PubMed: 22369928]

78. Wolf J, LoRusso PM, Camidge RD, Perez J, Tabernero J, Hidalgo M, et al. A phase I dose escalation study of NVP-BGJ398, a selective pan FGFR inhibitor in genetically preselected advanced solid tumors. Cancer Research. 2012; 72(8 Suppl):A122.

79. Qing J, Du X, Chen Y, Chan P, Li H, Wu P, et al. Antibody-based targeting of FGFR3 in bladder carcinoma and $\mathrm{t}(4 ; 14)$-positive multiple myeloma in mice. J Clin Invest. 2009; 119:1216-2129. [PubMed: 19381019] 
80. Bai A, Meetze K, Vo NY, Kollipara S, Mazsa EK, Winston WM, et al. GP369, an FGFR2-IIIbspecific antibody, exhibits potent antitumor activity against human cancers driven by activated FGFR2 signaling. Cancer Res. 2010; 70:7630-7639. [PubMed: 20709759]

81. Pai R, Dunlap D, Qing J, Mohtashemi I, Hotzel K, French DM. Inhibition of fibroblast growth factor 19 reduces tumor growth by modulating beta-catenin signaling. Cancer Res. 2008; 68:50865095. [PubMed: 18593907]

82. Tolcher A, Papadopoulos K, Agnew J, Marshall J, Tanzola C, Zanghi J, et al. Preliminary results of a phase 1 study of FP-1039 (FGFR1:Fc), a novel antagonist of multiple fibroblast growth factor (FGF) ligands. patients with advanced malignancies Molecular Cancer Therapeutics. 2009; 8(12 Suppl):A103. 


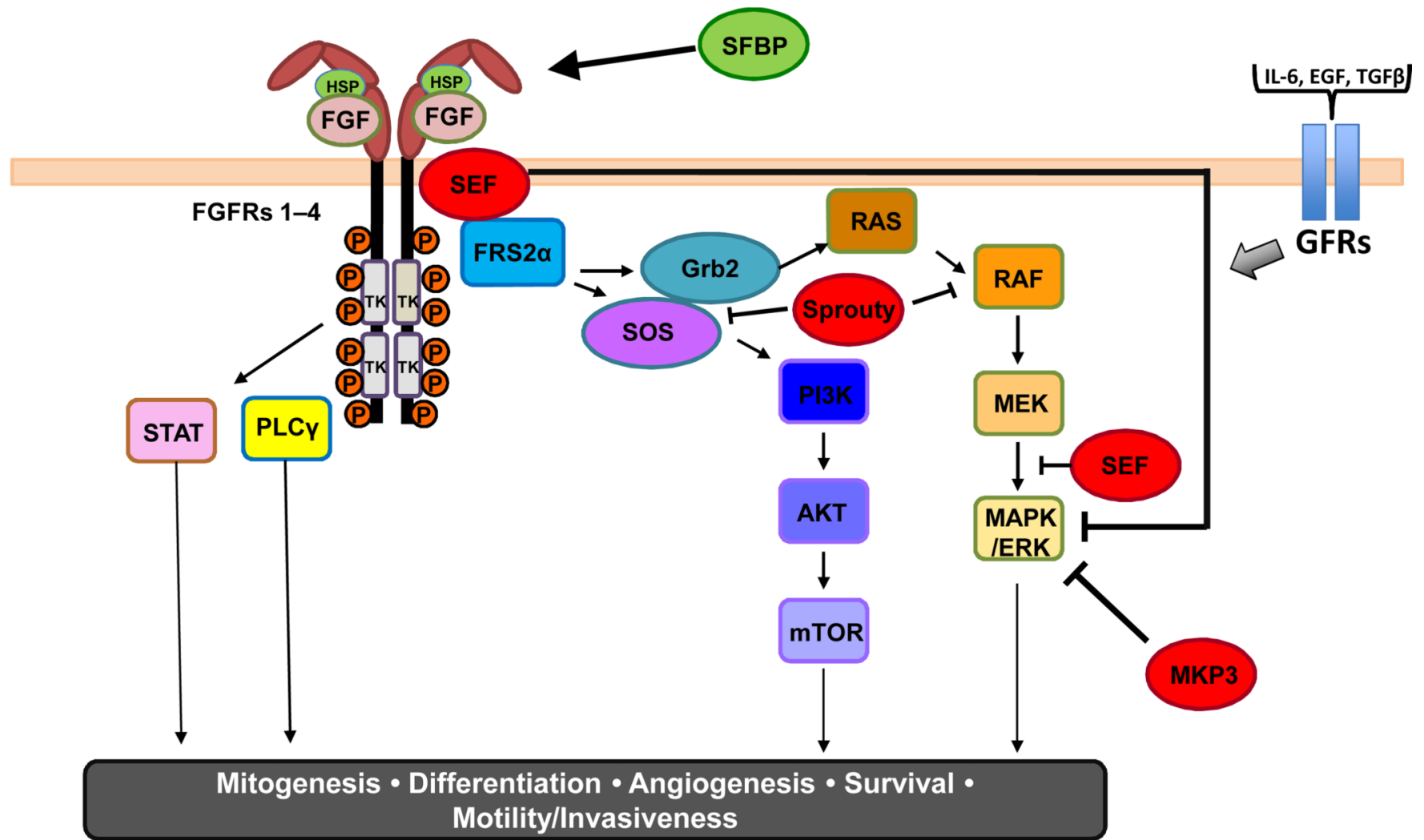

Figure 1.

The FGF/FGFR signaling pathway. FGF ligand binding triggers formation of the FGF/ FGFR/HSP complex, leading to autophosphorylation of the FGFR. Docking proteins such as FGFR substrate 2 (FRS2a) and phospholipase C gamma (PLC $\gamma$ ) activate downstream pathways, including RAS/RAF/MEK, phosphoinositide 3-kinase (PI3K)/AKT/mammalian target of rapamycin (mTOR), and signal transducer and activator of transcription. The pathway can be positively (HSP, SFBP) or negatively (SEF, Sprouty, MKP3) regulated at many different nodes. Intermediaries of FGF signaling are also activated by additional cytokines and growth factors (e.g. IL-6, EGF, and TGF $\beta$ ). 


\section{Mouse normal prostate epithelial cells (mNPE)/UGSM-GFP}

Kidney Caspule

Normal prostate-like glands
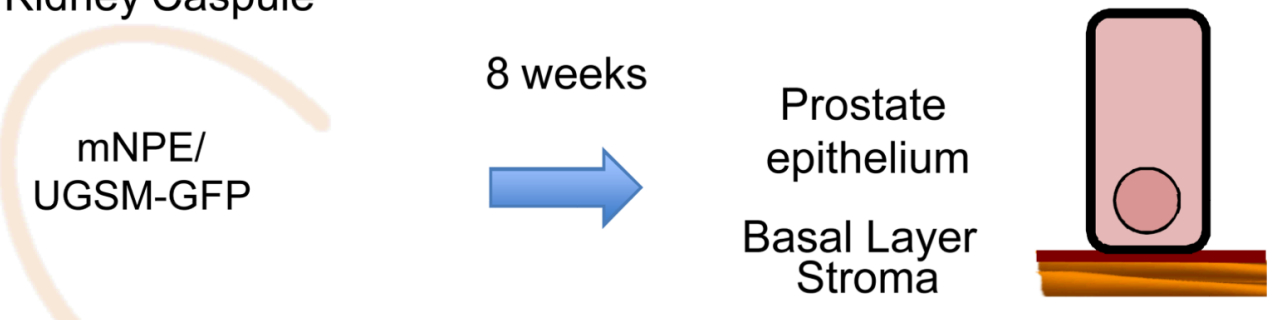

\section{Mouse normal prostate epithelial cells (mNPE)/UGSM-FGF10}

Kidney Caspule

Well differentiated

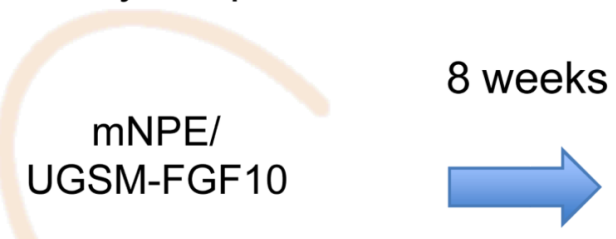

Prostate carcinoma

Normal prostate-like glands

Kidney Caspule

Mouse normal prostate epithelial cells (mNPE)-Dominant Negative FGFR1/UGSM-FGF10

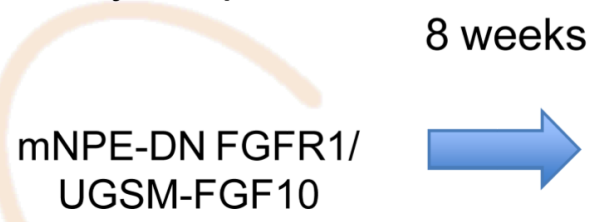
Prostate epithelium
Basal Layer
Stroma

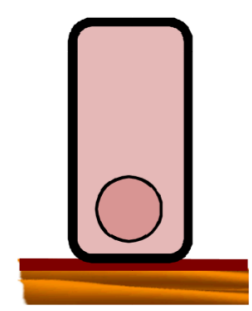

Figure 2.

Mesenchymal expression of FGF10 leads to the formation of FGFR1 dependent PIN or prostate cancer in a tissue recombination prostate regeneration system. When mouse normal prostate epithelial cells (mNPE) are mixed with embryonic urogenital sinus mesenchyme (UGSM) and grafted under the kidney capsule of mice, epithelial glands resembling the mouse prostate are formed. When mNPE are mixed with UGSM overexpressing FGF10, well differentiated prostate carcinoma develops and these cancer cells express higher androgen receptor levels than that observed in normal prostate-like glands. Inhibition of epithelial FGFR1 signaling using dominant-negative FGFR1 leads to reversal of the cancer phenotype. 
a. FGFR1 activation leads to PCa development and metastases (ref. 38)

JOCK-1 mice: mice expressing synthetic ligand-inducible FGFR1 (iFGFR1). iFGFR1 activation was induced by chemicals inducers of dimerization (CID)

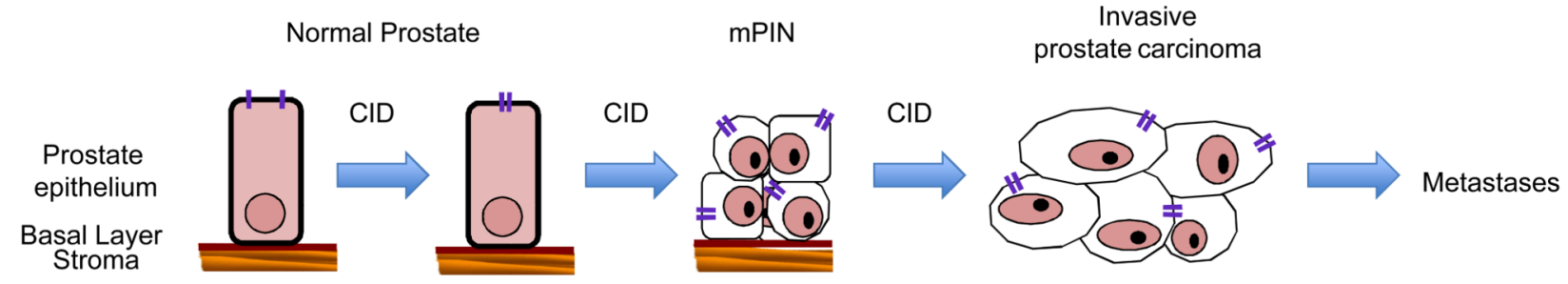

I liFGFR1

\section{b. Conditional deletion of FRS2 $\alpha^{\mathrm{Cn}}$ (FRS2 $\alpha^{\mathrm{Cn}}$ ) in TRAMP mice impairs PCa progression (ref. 39)}

TRAMP mice: transgenic adenocarcinoma of the mouse prostate (TRAMP) developed by expressing of SV40 T antigen in the mouse prostate

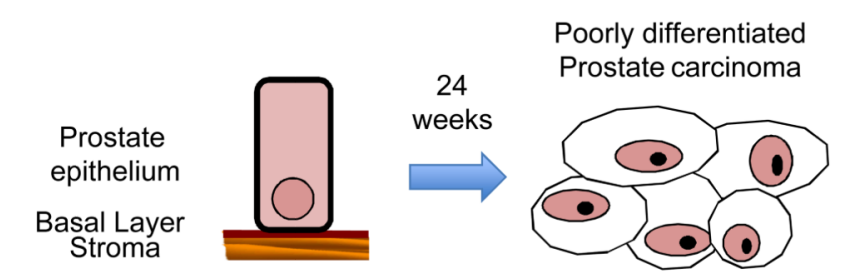

FRS $2 \alpha^{C n} / T R A M P$ hybrid: loxP-Cre recombination system was used to inactivate Frs $2 \alpha$ alleles in the prostate epithelium (FRS $\left.2 \alpha^{C n}\right)$ and these mice were crossed with TRAMP mice

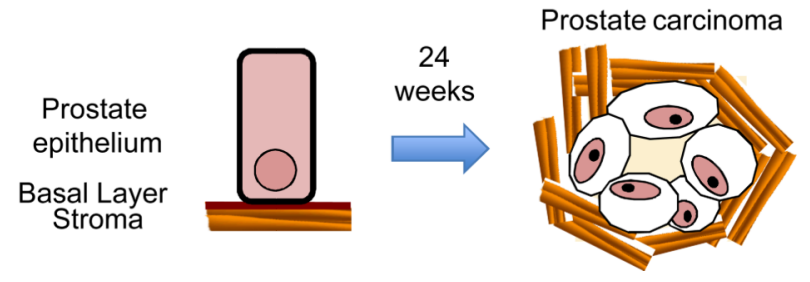

Figure 3.

Activation of FGFR axis mediates prostate cancer development and progression. A. Inducible FGFR1 (iFGFR1) prostate mouse model (named the juxtaposition of CID and kinase1 (JOCK1)). Activation of iFGFR1 with chemical inducers of dimerization (CID) led to PIN, invasive prostate cancer, and metastases. B, Transgenic adenocarcinoma of the mouse prostate (TRAMP) (established by expressing of SV40 T antigen in the mouse prostate) develop poorly differentiated prostate carcinomas at 24 weeks of age. Conditional deletion of FRS $2 a$ in the mouse prostate inhibited the initiation and progression of prostate cancer in the TRAMP model. 
a.

\section{Prostate cancer progression}

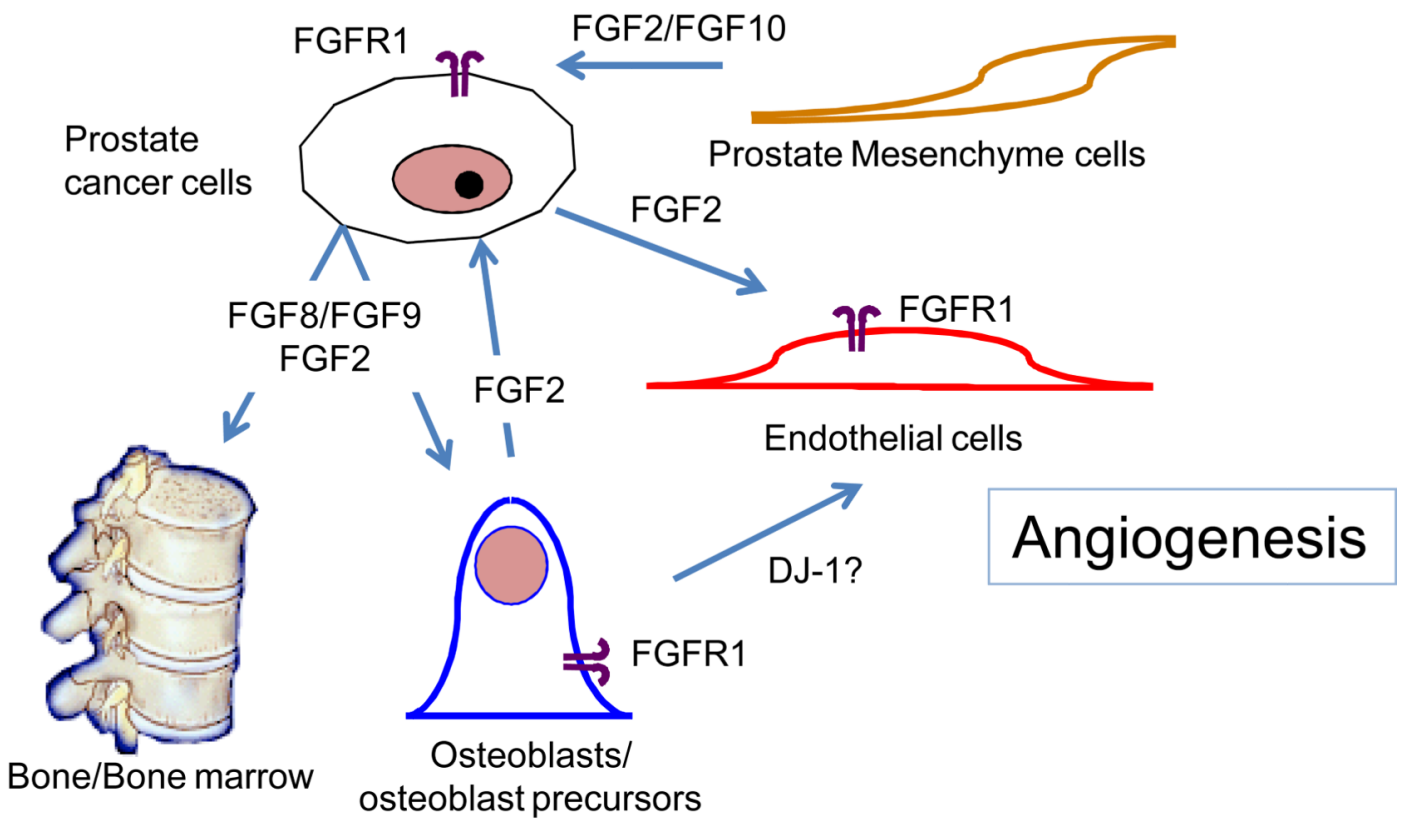

\section{Bone remodeling}


b

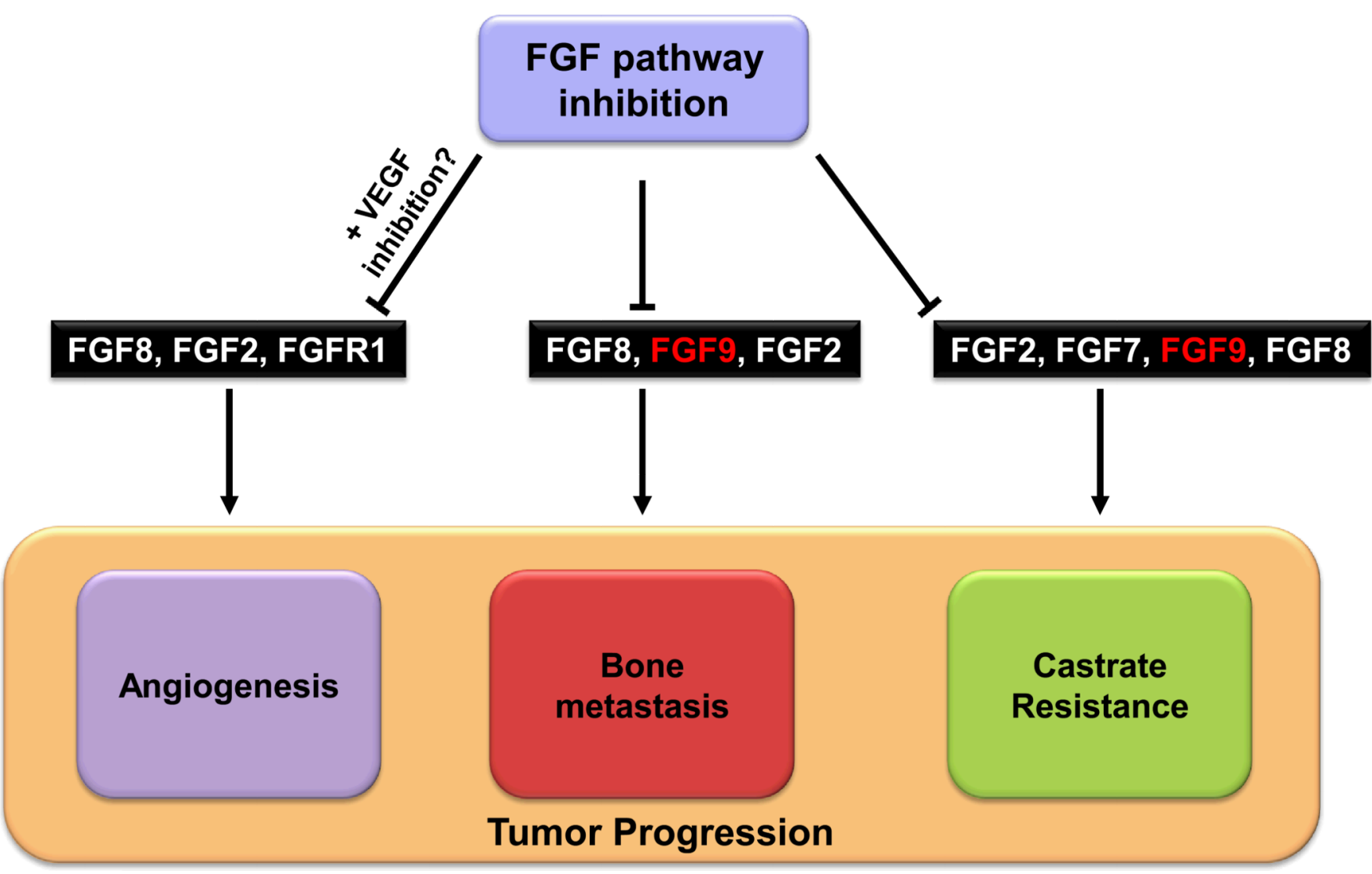

Figure 4.

A. A model of prostate cancer-stroma interaction mediated by FGFR and ligands. FGFR1 is expressed by prostate cancer cells, osteoblasts and endothelial cells. FGF2 is produced by prostate cancer cells and induces osteogenesis, bone remodeling, and angiogenesis. Angiogenesis in turn favors prostate cancer progression. Prostate mesenchymal cell production of FGF2/FGF10 activate FGFR1 in prostate cancer cells leading cancer progression. FGF8/FGF9 is produced by prostate cancer cells during growth in bone (bone metastases) and induces osteogenesis and bone remodeling. Activated osteoblasts induce angiogenesis. B. Inhibition of the FGF pathway can affect tumor progression by targeting multiple biological pathways. 


\section{FGF pathway inhibitors}

Table 1

Mechanism of action and clinical development stage of agents targeting the FGF pathway. Trials were identified from www.clinicaltrials.gov.

\begin{tabular}{|c|c|c|c|}
\hline Agent & Company & Target(s) & Clinical Development \\
\hline \multicolumn{4}{|c|}{ Small-molecule multikinase inhibitors } \\
\hline Dovitinib (TKI258) & Novartis & $\begin{array}{l}\text { FGFR1-3, VEGFR1-3, } \\
\text { PDGFR } \beta \text {, FLT-3, c-KIT }\end{array}$ & $\begin{array}{l}\text { Ongoing phase } 2 \text { trial in CRPC patients with } \\
\text { bone metastases(NCT00831792) } \\
\text { Phase } 3 \text { investigation in metastatic renal cell } \\
\text { carcinoma (NCT01223027) }\end{array}$ \\
\hline Nintedanib (BIBF1120) & Boehringer Ingelheim & $\begin{array}{l}\text { FGFR1-3, VEGFR1-3, } \\
\text { PDGFRa and }-\beta \text {, Src, Lck, Lyn }\end{array}$ & $\begin{array}{l}\text { Completed phase } 2 \text { trial in hormone-refractory } \\
\text { prostate cancer (NCT00706628) } \\
\text { Phase } 3 \text { investigation in NSCLC } \\
\text { (NCT00806819, NCT00805194) and ovarian } \\
\text { cancer (NCT01015118) }\end{array}$ \\
\hline Masitinib & AB Science & FGFR3, PDGFR, c-KIT & $\begin{array}{l}\text { No ongoing trialsspecifically in CRPC } \\
\text { Phase } 3 \text { investigation in GIST (NCT00812240, } \\
\text { NCT01694277), pancreatic cancer } \\
\text { (NCT00789633), multiple myeloma } \\
\text { (NCT01470131), and metastatic melanoma } \\
\text { (NCT01280565) }\end{array}$ \\
\hline Lenvatinib (E7080) & Eisai & $\begin{array}{l}\text { FGFR1, VEGF1-3, PDGFRa } \\
\text { and }-\beta, \mathrm{c}-\mathrm{KIT}\end{array}$ & $\begin{array}{l}\text { No investigation specifically in CRPC } \\
\text { Phase } 3 \text { investigation in thyroid cancer } \\
\text { (NCT01321554) }\end{array}$ \\
\hline Brivanib & Bristol-Myers Squibb & FGFR1, VEGFR2 & $\begin{array}{l}\text { No ongoing trials specifically in CRPC } \\
\text { Phase } 3 \text { investigation in liver cancer } \\
\text { (NCT00825955) and HCC (NCT00908752, } \\
\text { NCT01108705, NCT00858871) }\end{array}$ \\
\hline Orantinib (TSU-68) & Taiho Pharmaceuticals & $\begin{array}{l}\text { FGFR1, VEGFR2, PDGFR } \beta \text {, } \\
\text { KDR }\end{array}$ & $\begin{array}{l}\text { No ongoing trials specifically in CRPC } \\
\text { Phase } 3 \text { investigation in HCC(NCT01465464) }\end{array}$ \\
\hline PD173074 & (none) & FGFR1, VEGFR2 & Preclinical investigation \\
\hline \multicolumn{4}{|c|}{ Small-molecule tyrosine kinase inhibitors selective for FGF pathway } \\
\hline AZD4547 & AstraZeneca & FGFR1-3 & $\begin{array}{l}\text { No ongoing trials specifically in CRPC } \\
\text { Phase } 2 \text { investigation in breast cancer } \\
\text { (NCT01202591) and gastric cancer } \\
\text { (NCT01457846) }\end{array}$ \\
\hline AZD8010 & AstraZeneca & FGFR1-4 & Preclinical investigation \\
\hline BGJ398 & Novartis & FGFR1-3 & $\begin{array}{l}\text { Phase } 1 \text { trial in advanced solid tumors with } \\
\text { FGFR amplifications or } \\
\text { mutations(NCT01004224) }\end{array}$ \\
\hline \multicolumn{4}{|l|}{ Monoclonal antibodies } \\
\hline GP369 & AVEO Pharmaceuticals & FGFR2-IIIb isoform & Preclinical investigation \\
\hline MFGR1877S (R3Mab) & Roche/ Genentech & FGFR3 & $\begin{array}{l}\text { Phase } 1 \text { trial in advanced solid } \\
\text { tumors(NCT01363024) }\end{array}$ \\
\hline $1 \mathrm{~A} 6$ & Roche/ Genentech & FGF-19 ligand & Preclinical investigation \\
\hline \multicolumn{4}{|l|}{ Ligand trap } \\
\hline HGS1036 (FP-1039) & $\begin{array}{l}\text { GlaxoSmithKline, Human } \\
\text { Genome Sciences, Five } \\
\text { Prime Therapeutics }\end{array}$ & $\begin{array}{l}\text { FGF ligands that have affinity } \\
\text { for the FGFR 1-IIIc isoform }\end{array}$ & $\begin{array}{l}\text { Phase } 1 \text { trials in advanced solid tumors } \\
\text { (NCT00687505, NCT01604863) }\end{array}$ \\
\hline
\end{tabular}

FLT-3, fms-related tyrosine kinase-3; GIST, gastrointestinal stromal tumor; KDR, kinase insert domain receptor; NSCLC, non-small cell lung cancer. 